\title{
Diffusion in spatially and temporarily inhomogeneous media: Effects of turbulent mixing
}

\author{
H. Lehr ${ }^{*}$ and F. Sagués \\ Departament de Química Física, Universitat de Barcelona, 08028 Barcelona, Spain \\ J. M. Sancho \\ Departament d'Estructura i Constituents de la Materia, Universitat de Barcelona, 08028 Barcelona, Spain
}

(Received 28 January 1997)

\begin{abstract}
We consider diffusion of a passive substance $C$ in a phase-separating nonmiscible binary alloy under turbulent mixing. The substance is assumed to have different diffusion coefficients in the pure phases $A$ and $B$, leading to a spatially and temporarily dependent diffusion "coefficient" in the diffusion equation plus convective term. In this paper we consider especially the effects of a turbulent flow field coupled to both the Cahn-Hilliard type evolution equation of the medium and the diffusion equation (both, therefore, supplemented by a convective term). It is shown that the formerly observed prolonged anomalous diffusion [H. Lehr, F. Sagués, and J.M. Sancho, Phys. Rev. E 54, 5028 (1996)] is no longer seen if a flow of sufficient intensity is supplied. [S1063-651X(97)00108-6]
\end{abstract}

PACS number(s): 47.27.Sd, 64.60.My

\section{INTRODUCTION}

In a recent publication [1] (hereafter referred to as I) we treated diffusion in a special inhomogeneous medium. We were then interested in finding out how the time dependence of the variance of a passive scalar field immersed in a binary alloy changes when coupled to the spatial and temporal inhomogeneities of the underlying phase-separating medium.

In this paper, we will consider a similar model but include turbulent mixing. In [2,3] we have been concerned with the diffusion of a passive scalar convected by a random incompressible flow using the analytical and simulation techniques of stochastic differential equations. The role of the medium was played by the mentioned turbulent flow, which is represented mathematically by a two-dimensional, isotropic, stationary, homogeneous, and incompressible velocity field. In [4] it has been shown how to generate such a vector field in an efficient manner. The latter ansatz has now been extended to explicitly incorporate viscosity [5].

We have opted to use, as a medium, a solution of a dynamical equation corresponding to a phase segregation problem, i.e., in this case the well-known Cahn-Hilliard equation [6] supplemented by a convective term [7] (see, e.g., [8-10] for some newer literature on the theoretical and [11-13] on the applied aspects of this subject),

$$
\frac{\partial \chi}{\partial t}=\nabla^{2}\left(-\chi+\chi^{3}-\nabla^{2} \chi\right)-\vec{\nabla} \cdot(\vec{v} \chi),
$$

where $\vec{v}(\vec{r}, t)$ is a stochastic, isotropic, and incompressible vector field that is our model of turbulent stirring. The initial distribution $\chi(r, 0)$ is chosen to be

$$
\chi(r, 0)=\chi_{0}+\alpha
$$

\footnotetext{
*Permanent address: I. N. Stranski-Institut, Sekretariat ER 1, Technische Universität Berlin, Straße des 17. Juni 112, D-10623 Berlin, Germany.
}

$\alpha$ is a (uniform) random variable, whose actual range is not of critical importance as long as its average vanishes. Here we have chosen $\alpha \in[-0.1,0.1]$.

This equation describes the phase separation following a quench of a nonmiscible binary alloy (with phases $A$ and $B$ ) inside its coexistence curve. It is known (see, e.g., [7] and references therein) that the solutions to this equation are, depending on the intensity of the turbulence, more or less inhomogeneous. In the zero intensity limit this medium is very structured, its configuration depending on the relative concentration of the phases. Raising the intensity of the turbulent flow the structures, quite naturally, get distorted and less regular. In the high intensity limit, phase separation even stops and we arrive at a homogeneous mixture of the participating phases. For more details see [7] and also I.

The velocity field appearing in Eq. (1) has a zero mean and is, for simplicity, Gaussian correlated

$$
\left\langle v^{i}\left(\vec{r}_{1}, t_{1}\right) v^{j}\left(\vec{r}_{2} \cdot t_{2}\right)\right\rangle=R^{i j}\left(\left|\vec{r}_{1}-\vec{r}_{2}\right|,\left|t_{1}-t_{2}\right|\right),
$$

where $v^{i}, v^{j}$ with $i, j \in\{x, y\}$ stand for the components the two-dimensional velocity field. This vector field is constructed using the stream function $\eta$

$$
\vec{v}=\left(-\frac{\partial \eta}{\partial y}, \frac{\partial \eta}{\partial x}\right)
$$

which in turn follows the Langevin equation

$$
\frac{\partial \eta}{\partial t}=\nu \nabla^{2} \eta(\vec{r}, t)+Q\left[\lambda^{2} \nabla^{2}\right] \vec{\nabla} \cdot \vec{\xi}(\vec{r}, t)
$$

(here the dynamical viscosity $\nu$ appears explicitly [5]). $\vec{\xi}$ represents herein a stochastic noise term, whose components are spatially and temporarily $\delta$ correlated.

$$
\left\langle\xi^{i}(\vec{r}, t) \xi^{j}\left(\vec{r}^{\prime}, t^{\prime}\right)\right\rangle=2 \epsilon_{0} \nu \delta\left(\vec{r}-\vec{r}^{\prime}\right) \delta\left(t-t^{\prime}\right) \delta^{i j} .
$$


It can be shown that this vector field $\vec{v}$ exhibits in dependence of the operator $Q[\cdots]$ the energy spectra characteristic for turbulence (e.g., that of Kraichnan [14], which will solely be used in our calculations, or that of KarmanObukhov [15]).

The turbulent flow is characterized by the parameters $\epsilon_{0}$ which represents the intensity of the noise source divided by the viscosity, $\lambda$ which is connected to the correlation length $l_{0}$ of the flow by

$$
l_{0}=\frac{\sqrt{\pi}}{2} \lambda \text {, }
$$

(for the Kraichnan type of energy spectrum; see [5]), and by the dynamical viscosity $\nu$, which together with $\lambda$ defines the correlation time $t_{0}$ of the flow

$$
t_{0}=\frac{\lambda^{2}}{\nu}
$$

again for Kraichnan's spectrum. The intensity of the turbulence, additionally, is given by

$$
u_{0}^{2}=\frac{\epsilon_{0}}{8 \pi \lambda^{4}}
$$

Diffusion of the substance $C$ is now treated via the usual diffusion equation, again supplemented by a convective term using the same velocity field as in Eq. (1). As a coupling between the phase-separating medium and the diffusion we have simply chosen

$$
D(\vec{r}, t)=[1-a \chi(\vec{r}, t)] D^{*}
$$

where $D^{*}$ is a value of input that together with the parameter $a$ (here: $a=0.85$ ) defines the diffusion constants in the pure phases. Again we refer to I for a discussion of this coupling and its parameters.

So, let $\psi(r, t)$ be a passive, i.e., nonreacting, scalar field, that describes the density of the substance $C$. Then we have

$$
\frac{\partial \psi}{\partial t}=\vec{\nabla} \cdot[D(\vec{r}, t) \vec{\nabla} \psi(\vec{r}, t)]-\vec{\nabla} \cdot[\vec{v} \psi(\vec{r}, t)],
$$

where the second term on the right hand side again describes convection by the same turbulent vector field, as mentioned above.

The object of our interest now is the variance of $r$ of this scalar field with time, i.e.,

$$
\sigma(t)=\left\langle(\Delta r)^{2}\right\rangle=\left\langle r^{2}\right\rangle-\langle r\rangle,
$$

where $\langle f(r)\rangle$ is defined as the average of $f(r)$ over different realizations of $\psi$ [and therefore as the average over different realizations of the turbulent field, as well as that of the "medium,' that is being represented by $\chi(r, t)$ and is, because of the random nature of the initial conditions, also a stochastic process).

As is well known, in homogeneous media this dispersion grows in the long time limit linearly with time, the proportionality constant being four times (in two-dimensions) the effective diffusion constant $D_{\text {eff }}$

$$
\sigma(t)=4 D_{\mathrm{eff}} t
$$

whose value can be calculated analytically for small intensities of the turbulent advective field [2,3]. For the sake of easily identifying diffusional regimes we defined in I

$$
D(t)=\frac{\sigma(t)}{4 t},
$$

which was then usually represented and discussed.

Additionally, we derived in I an expression for the time dependence of $D(t)$ for the case of an unstirred phase separation. Its counterpart for the present problem can be evaluated similarly as

$$
\begin{aligned}
D(t)= & D^{*}\left(1-a \chi_{0}\right)-\frac{a D^{*}}{2 t} \\
& \times \int_{0}^{t} d t \iint d x d y\left\langle\left(x \frac{\partial \chi}{\partial x}+y \frac{\partial \chi}{\partial y}\right) \psi\right\rangle_{\chi, \vec{v}} \\
& +\frac{1}{2 t} \int_{0}^{t} d t \iint d x d y\langle\vec{r} \cdot \vec{v} \psi\rangle_{\chi, \vec{v}}, \\
\equiv & \langle D\rangle_{r, \chi}-\int_{0}^{t} \delta D(t) d t+\Delta D(t),
\end{aligned}
$$

which now has been supplemented by an additional term $\Delta D(t)$ due to the presence of the turbulent field. [Subscripts on the angular brackets indicate whether averaging is over space $(r)$, or over realizations of $\chi$ or the flow $\vec{v}$.] For homogeneous media this term $\Delta D$ has been evaluated analytically in the limit of small correlation times of the flow [2,5]. Note though, that here, i.e., with an inhomogeneous and dynamically evolving medium, $\Delta D$ depends generally on time.

One of the main results of I was that diffusion in our phase-separating medium without stirring was anomalous. Indeed, we gave evidence that $D(t)$ goes like $t^{-1 / 3}$ to a constant final value $D_{\infty}$. This is an extremely slow process. Analyzing the results with the aid of some analytical treatment, we suggested this to be due to a "reservoir effect"' and that the total interface length would be an adequate measure for it. Under a "reservoir effect" we understand the following: In the regions with a low diffusion coefficient it takes a very long time for the matter to diffuse through these regions. Therefore, even at longer times, a good part of the dispersed matter will be collected in exactly these regions, thus giving rise to an ever decreasing $D(t)$. Moreover, these regions have their own dynamics, which gives a process that will only asymptotically reach a constant value for $D(t)$. Since the integral representing $\delta D$ gets its contributions only from the interface region, we concluded that the total interface length was the most natural way to measure the reservoir effect. Interchangeably, we can investigate the effect on the linear (characteristic) size of the growing pure-phase domains, since these two measures are not independent of each other.

Looking now at the model we want to investigate here, one realizes that $D(t)$ couples both directly and indirectly to the random flow. Directly due to the presence of a new term in Eq. (8) and indirectly due to the dependence on the un- 
derlying structuring of $\chi$. Let us therefore first treat the effect that such a random flow has on $\chi$ and even more importantly on $L(t)$, the total length of the interface (or rather the total area; see below).

In view of the rather numerous parameters involved in the model (the diffusion coefficient $D^{*}$, the coupling constant $a$, the mean composition $\chi_{0}$, the noise strength $\epsilon_{0}$, the viscosity $\nu$, and the parameter $\lambda$, which is connected with the correlation length of the flow) we have fixed both the average diffusion coefficient $\langle D\rangle=D^{*}\left(1-a \chi_{0}\right)=0.5$, the coupling parameter $a$, and the mean composition $\chi_{0}$ (see, though, below), which normally was chosen to be zero (or, equivalently, we consider equipartial or critical mixtures).

For the sake of brevity, and in order to avoid repetitions, we do not wish to dwell on numerical details but on the necessary ones. The Cahn-Hilliard equation as well as the diffusion equation have been discretized according to standard procedures; see I for more details. As to the flow, the numerical aspects have been discussed in some detail in [5]. Here it may suffice to say that Eq. (3) can be solved exactly in Fourier space and that thus the obtained solution will then be Fourier antitransformed and used in both Eqs. (1) and (5).

The numerical errors of our results, equally that of the total interface area as well as that of $D(t)$, are extremely difficult to estimate, also for reasons of computer time. In I we estimated the error for $D(t)$ in the case without flow and from our experience we know that here the errors are not significantly higher (i.e., they will be of the order of 10$15 \%)$. "Real" error estimates or even bounds, though, are not available.

\section{INFLUENCE OF THE FLOW ON THE INTERFACE LENGTH}

In [7] some of us have already been concerned with the influence of a turbulent flow on the solutions of the CahnHilliard equation plus convective supplement. We were then more interested in the temporal dependence of the spatial extension of the appearing structures than in the interface length. The main result of [7] can be summarized as follows: for low and medium intensities of the flow the linear structure size grows in the long time limit according to the Lifshitz-Slyozov behavior; for smaller times, a different growth law has been observed [7]. Raising the intensity though, one finds a limit intensity beyond which the system supports only structures of a certain size; those larger get ruptured by the flow. It was suggested in [7] that for every $u_{0}$ there exists a critical $\lambda_{c}$, such that for every value $\lambda>\lambda_{c}$, one will find frozen growth. In particular, it was found that

$$
\lambda_{c}^{2} \approx \frac{\pi^{2}}{2} \frac{\Sigma}{u_{0}}
$$

where $\Sigma=2 \sqrt{2} / 3 \approx 1$ is related to the surface tension.

In order to understand the above relation between the spatial correlation length of the flow and the question of frozen or sustained growth, it seems worthwhile to discuss the mixing effectivity-obviously a closely related concept-in dependence of the three parameters $u_{0}^{2}, l_{0}$, and $t_{0}$ of the flow. Qualitatively speaking, the mixing effectivity is best with

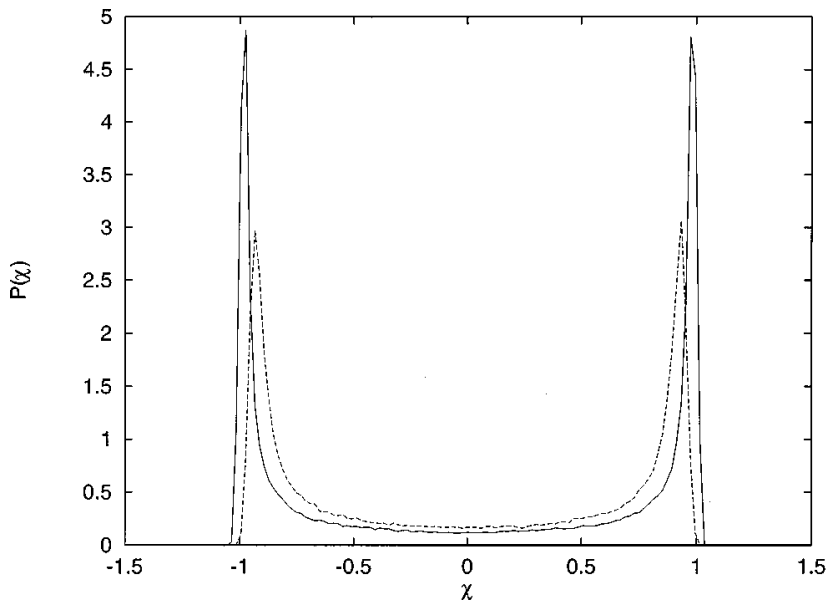

FIG. 1. Representation of the distribution of $\chi$ at $t=500$ for two different values of the flow intensity. (Solid line: $u_{0}^{2}=0$, broken line: $u_{0}^{2}=0.0870$.) In the mixed case the interface gets sensibly less sharp, so that a "zero" width assumption is no longer valid. See text for details. (Grid size $N=128, \chi_{0}=0.0, \lambda=2$, and $\nu=1,10$ realizations).

small eddies of high intensity and long correlation time. The proportionality to the intensity needs no further explanation. The dependence on $l_{0}$ may be understood from an extreme case: consider one sole circular eddy of the size of the system (very large $l_{0}$ ) centered at the origin. Instead of mixing the phases, this would just give the whole system an angular velocity, depending on the intensity. The mixing effectivity would be (close to) zero. For smaller eddies, on the other hand, there is always an exchange between the eddies, leading to mixing, if their correlation time is not too short. If we have many small eddies but with a very short correlation time, again there will be little mixing, because there will be very little exchange between the eddies. (In the opposite case of very long correlation times, one will find a constant flow between the eddies, which implies a rather effective mixing.)

A last preparatory remark may be in place: since the average composition $\chi_{0}$ is conserved in the Cahn-Hilliard equation (with and without convection) the number of structures times their respective area must be constant. From this follows that for a growing spatial extension of the structures, their number must decrease (one observes that smaller structures are unstable and vanish in favor of the larger ones). Since their number now is antiproportional to the square of the linear dimension, the total interface length (which is proportional to the number and the linear extension) will decrease with time. In the undisturbed problem without mixing we could simply define

$$
L(t)=\left\langle\iint d x d y\left(1-\chi^{2}\right)\right\rangle_{\chi} .
$$

Because of the pronounced sharpness of the interface (see Fig. 1) this area was practically identical to the total interface length. We found in I that $L(t) \propto t^{-1 / 3}$. In the disturbed case with mixing the interface now gets sensibly broader. In Fig. 1 we show the (normalized) distribution of $\chi$ at time $t=500$ for zero noise and for $u_{0}^{2}=0.0870$. Since it is evident from Fig. 1 that the above definition will not yield the inter- 
face length, but rather the total interface area, some care has to be taken. We therefore contrasted our results by calculating also the characteristic linear size of the structures. This is evaluated as usual from the circular average $g(r, t)$ of the correlation function

$$
G(\vec{r}, t)=\left\langle\frac{1}{N^{2}} \sum_{r^{\prime}}\left[\chi\left(\vec{r}+\vec{r}^{\prime}, t\right) \chi\left(\vec{r}^{\prime}, t\right)-\chi_{0}^{2}\right]\right\rangle_{\chi, \vec{v}},
$$

(where $N$ is the size of the system). The "characteristic" size $R(t)$ of the structures is now determined by the first zero of the function $g(r, t)$ with respect to $r$. Calculations of this type, though, yield for the time range considered here results that are relatively independent of the noise intensities used. This is because we are still in the coarsening regime [7], i.e., the dominant process is still the coalescence of droplets rather than their being moved around by the flow. This is also expressed by the fact that we do not find a growth proportional to the Lifshitz-Slyozov $t^{1 / 3}$ law.

In view of the results concerning $D(t)$ to be presented later on and realizing also from Eq. (8) that the "indirect" term gets contributions from the whole interfacial area, the more consistent measure for our time range is $L(t)$, as defined above. We will hence base our argumentation on the interfacial area.

We have calculated $L(t)$ for different choices of the parameters $\epsilon, \lambda$, and $\nu$. One finds the following results.

(a) The interface area decreases in a slower manner with a growing intensity of the random flow [see Fig. 2(a)]. Mixing is therefore more effective.

(b) The interface area decreases faster with growing $\nu$, i.e., decreasing correlation time.

(c) The same is true for growing $\lambda$. Changing $\lambda$, though, changes all $u_{0}^{2}, l_{0}$, and $t_{0}$. In view of the importance of the intensity, changing just $\lambda$ is inconclusive.

(d) For a given intensity of the random flow $u_{0}^{2}$, the temporal development of the interface is (almost) independent of the different possible choices of $\epsilon_{0}$ and $\lambda$ [see Fig. 2(b)]. Note that here-as in (c) - $t_{0}$ is changed. Additionally keeping $t_{0}$ constant [inset of Fig. 2(b)], the results are statistically equal.

Results (d) might at first sight be a little unexpected, keeping in mind that according to Eq. (9) raising $\lambda$ for constant $u_{0}^{2}$ we are going in the direction of frozen growth. They show, though, that for $L(t)$ the most important parameter is the intensity of the turbulent flow.

We have also calculated the equal point correlation function of $\langle\chi(\vec{r}, t) \chi(\vec{r}, t+s)\rangle_{r, \chi, \vec{v}}$ as a measure of the change in structure. The qualitative results are the same as above. In Fig. 3 we show this nonstationary correlation function for three values of the noise parameter $\epsilon_{0}=0,20$, and 35. Initially one finds for the $t$ chosen an increasing correlation, which is essentially due to the fact that at very short times the solutions of the Cahn-Hilliard equation have not yet reached their equilibrium values of \pm 1 . So, typically, one finds that initially the "bulk" values grow until they reach said values. The much more interesting and relevant information here is naturally the decay of the correlation. As to be expected, the correlation decays much more rapidly when the phase mixture is "stirred."
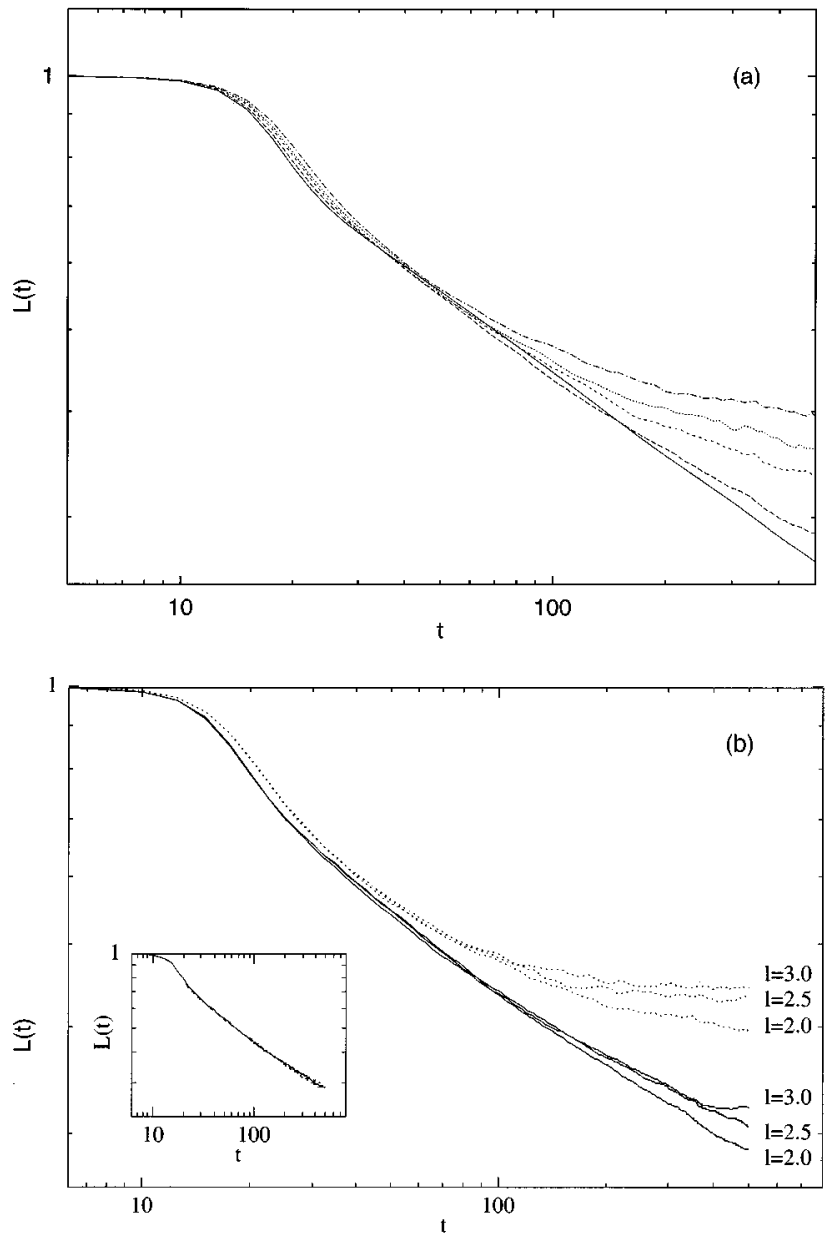

FIG. 2. Double-logarithmic representation of the total interface area $L(t)$ in dependence of time as a function of the parameters of the flow. (a) Varying the intensity $u_{0}^{2}$ of the flow $u_{0}^{2}$. (Solid line: $u_{0}^{2}=0.0000$, long dashes: $u_{0}^{2}=0.0249$, short dashes: $u_{0}^{2}=0.0497$, dots: $u_{0}^{2}=0.0622$, and dot dashes: $u_{0}^{2}=0.0807 ; \lambda=2, \nu=1$.) In (b) we show results for two constant intensities of the flow $u_{0}^{2}=0.0249$ (solid lines) and 0.0870 (dashed lines) varying both $\epsilon_{0}$ and $\lambda(\nu=1)$. Note that $t_{0}$ varies here. In the inset of (b) results for constant $u_{0}^{2}=0.0249$ and constant $t_{0}=4$ are shown. (Solid line: $\lambda=2.0, \quad \nu=1$, long dashes: $\lambda=2.5, \nu=1.5625$, short dashes $\lambda=3.0, \quad \nu=2.25$.) See text for details. (Grid size $N=128$, $\chi_{0}=0.0,10$ realizations.)

Summarizing, one can say that the total interface area is a continuous function of the parameters of the flow. Not only very near the limit for frozen growth, but also further away the time dependence of $L(t)$ changes considerably with the intensity of the flow. While correlation time does have an influence, though of second order, correlation length seems to play a minor role.

\section{INFLUENCE OF THE FLOW ON THE DIFFUSION OF THE SCALAR}

After having discussed in some detail the influence of a turbulent flow on the structurization of the underlying medium, we now proceed to discuss diffusion in such a dynamically evolving medium. In I we gave evidence to the fact that the interface in an undisturbed problem plays a major role. In fact, we found-as mentioned earlier-that the temporal de- 


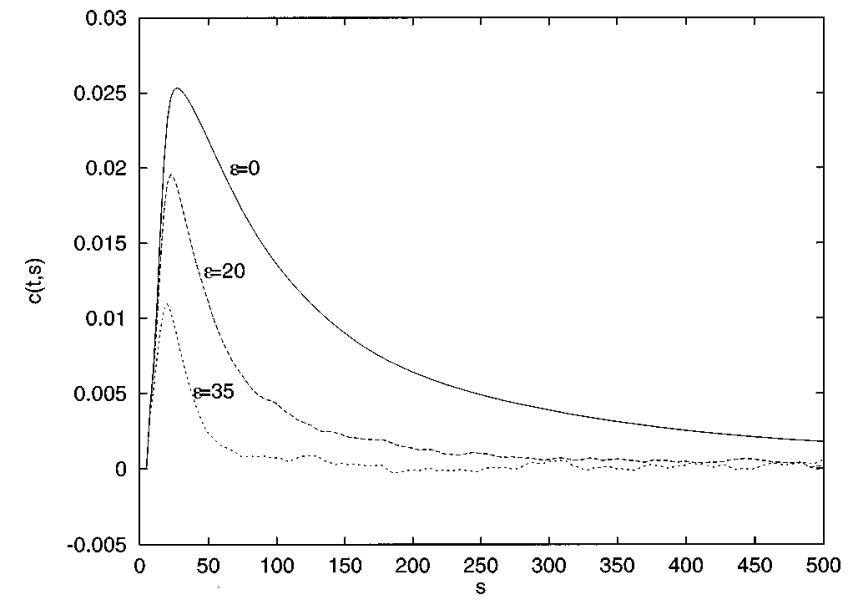

FIG. 3. The nonstationary correlation function $c(t, s)=\langle\chi(\vec{r}, t) \chi(\vec{r}, t+s)\rangle_{r, \chi, \vec{v}}$ for $\epsilon_{0}=0$ (solid line), $\epsilon_{0}=20$ (long dashes), and $\epsilon_{0}=35$ (short dashes) as a function of the time difference. ( $t=6.25$ was chosen such that the initial structurization $\chi$ has advanced considerably; $N=128, \lambda=2, \nu=1 ; 10$ realizations.)

velopment of the total interface length determines how $D(t)$ approaches its asymptotic value (which we calculated using the first order effective medium approximation; for the latter see, e.g., [16]). Now including a turbulent flow field, the analysis of I changes considerably. As described above and in I, the cause for the very prolonged abnormal diffusion was the "reservoir effect" which in turn depends heavily on the absence of a transport mechanism other than pure diffusion.

In Fig. 4 we show results for $D(t)$ corresponding to different intensities of noise $u_{0}^{2}$. Apart from finite size effects we find, for already rather weak noises, a diffusional regime. Quite expectedly, mixing has a destructive influence on the behavior described in I. In order to estimate the efficiency of the mixing, we also calculated $D(t)$ for the same parameters,

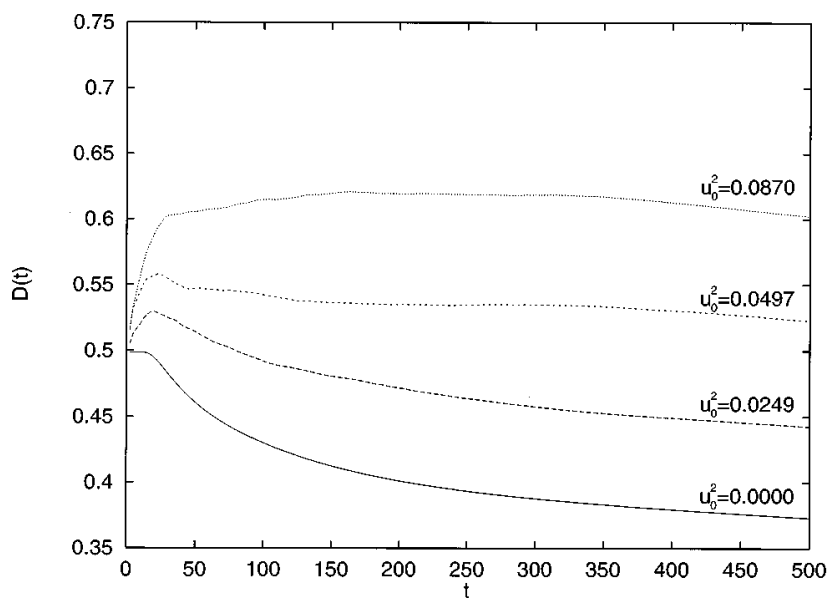

FIG. 4. Temporal development of $D(t)$ for different intensities of the flow $(\lambda=2, \nu=1)$. Even for relatively small intensities of the flow, the prolonged abnormal diffusion observed for $u_{0}^{2}=0$ is not found anymore. A diffusional regime is reached within the calculation time. Note, however, that for the constant $\langle D\rangle=D^{*}(1$ $\left.-a \chi_{0}\right)=0.5$ this depends on the average composition. The final dropping of the curves is due to finite size effects. (Results averaged over 10 runs with system size $N=128$.)
TABLE I. Estimate of effective differential diffusion constants. $\Delta D^{\mathrm{H}}$ was evaluated by numerical simulation putting $a=0$ which is equivalent to obtaining it from a homogeneous medium with $D=0.5$ [see Eq. (4)]. $\Delta D^{\mathrm{I}}$ was estimated from the data shown in Fig. 4 for the case of zero average composition. Although the medium is inhomogeneous and dynamically evolving, we do reach a diffusional regime within calculation time and therefore $\Delta D^{\mathrm{I}}$ can be estimated. (The size of the system was $N=128$, and the average was made over 10 runs up to $t=500$ or 20000 time steps; $\chi_{0}=0$, $\lambda=2$, and $\nu=1$.)

\begin{tabular}{lccc}
\hline \hline$\epsilon_{0}$ & $u_{0}^{2}$ & $\Delta D^{\mathrm{H}}$ & $\Delta D^{\mathrm{I}}$ \\
\hline 20 & 0.0497 & 0.108 & 0.035 \\
25 & 0.0622 & 0.133 & 0.070 \\
30 & 0.0746 & 0.158 & 0.103 \\
35 & 0.0870 & 0.183 & 0.119 \\
\hline \hline
\end{tabular}

but putting $a=0$. This gives a homogeneous medium with the diffusion coefficient equal to the average diffusion coefficient of the inhomogeneous problem (in the case of $\chi_{0}=0$ ). We could not estimate $\Delta D^{\mathrm{H}}$ (the deviation of the computed diffusion constant from the average diffusion constant in the homogeneous case) from the analysis made in [5], because here we are not in the limit of very short correlation times of the flow. Approximate results obtained by numerical simulation [simply putting $a=0$ in Eq. (4)] are shown in Table I. From there and Fig. 4 we can see that even with an intensity of the flow that in a homogeneous medium would result in $20 \%$ elevation of the effective diffusion constant only, the abnormal behavior found without mixing is not observed anymore. [In our numerical simulations we found diffusional behavior for $u_{0}^{2} \geqslant 0.0497(\lambda=2, \nu=1)$.] Turbulent mixing seems to be very efficient in this respect. In Table I we also show the numerical estimate of the effective diffusion constant for this scenario. We see that even in the case of higher intensity of the flow, the effective diffusion constant is considerably lower than it was for a homogeneous medium with the same characteristics. For very high intensities, though, we can expect the two values to coincide. A very rough estimate based on the linear dependence of both $\Delta D^{\mathrm{H}}$ and $\Delta D^{\mathrm{I}}$ (inhomogeneous case) says that for values above $u_{0}^{2}=0.16$ (for $\nu=1$; see below) we may expect the two to coincide. (One has to take into account, though, that higher intensity stirring stops phase separation, so that possibly this will be reached even earlier.)

We have also calculated $D(t)$ for two other average compositions $\chi_{0}= \pm 0.4$ as in I. This might have been interesting, because in those cases we found droplets instead of lamellar structures. The results, though, are-again as in I-strictly analogous to the ones presented in Fig. 4, albeit in some cases we had even more problems with finite size effects.

As we mentioned in the Introduction, the diffusing field $\psi$ couples both directly and indirectly to the flow. In order to estimate the influence of these two different couplings, we performed calculations neglecting either one of them. In Fig. 5 we show the corresponding results of calculating $D(t)$ (a) without any coupling or, equivalently, for $u_{0}^{2}=0$ [solid line], (b) dropping the convective term in (5) [long dashes], (c) dropping the convective term in (1) [short dashes], and (d) taking both couplings into account [dots]. The results shown, 


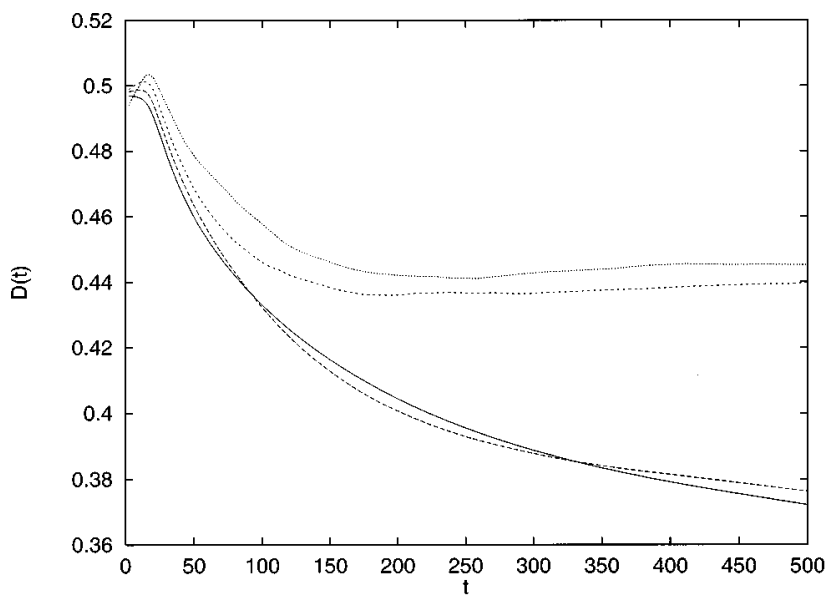

FIG. 5. Temporal development of $D(t)$ for different couplings to the flow. Solid line shows results for $u_{0}^{2}=0$ or, equivalently, no coupling. The long dashed line was obtained calculating $D(t)$ by dropping the convection term the differential equation for $\psi$. The short dashed line was obtained in a similar manner, only this time dropping the convection term in the Cahn-Hilliard type equation with convection. Finally, the dotted line includes both couplings. $\left(u_{0}^{2}=0.0497, \lambda=2, \nu=1\right.$, and $\chi_{0}=0$. System size was $N=128$, and results were averaged over 10 runs.)

and those for higher intensities of the flow, indicate that the indirect coupling is of minor importance. In effect, although there is a dependency of the results on the intensity also for the case of dropping the direct coupling, calculating $D(t)$ with only the convective term in the evolution equation for $\psi$, yields statistically the same results as in Fig. 4. In spite of the fact that, physically speaking, dropping one of the convection terms is not very sensible, it shows us that the transport of matter due to the mixing really is very effective. The important role of the underlying inhomogeneous medium in imposing a non-normal diffusion due to the formation of reservoirs is strongly reduced by adding a turbulent flow to the problem.

Let us now proceed to discuss the dependency of the different parameters involved. Apart from the dependency on $D^{*}$ and $\langle D\rangle$ already discussed in $\mathrm{I}$, for the dependency on the parameters of the flow, we can adopt exactly the same position as in the preceding section, where we discussed the influence on the total interface area. As to varying $\nu$ [and $\lambda]$, we find strictly the same behavior as above: since the mixing effectivity is proportional to the correlation time (and antiproportional to the correlation length), diminishing $\nu$ [or $\lambda$ ] we observe a transition from anomalous to diffusional behavior. (Let us remark once more, though, that changing only $\lambda$ is of lesser interest, because besides the correlation length, both intensity and correlation time are also changed.)

There is, though, a distinction in the relevance of the correlation time. This can be seen in Figs. 6(a) and 6(b). In Fig. 6(a) we show $D(t)$ for constant $u_{0}^{2}=0.0249$, analogous to Fig. 2(b), i.e., with constant $\nu$ and therefore variable $t_{0}$. In the preceding section we showed that the interface area depends very little on $\left(\epsilon_{0}, \lambda\right)$, as long as $u_{0}^{2}$ is constant. Here the case is very different. In fact, we again observe a transition from anomalous to diffusional behavior, in spite of the fact that $\lambda$ grows. If we compare this with Fig. 6(b), where also $t_{0}=4$ is kept constant, the reason seems to be clear. In
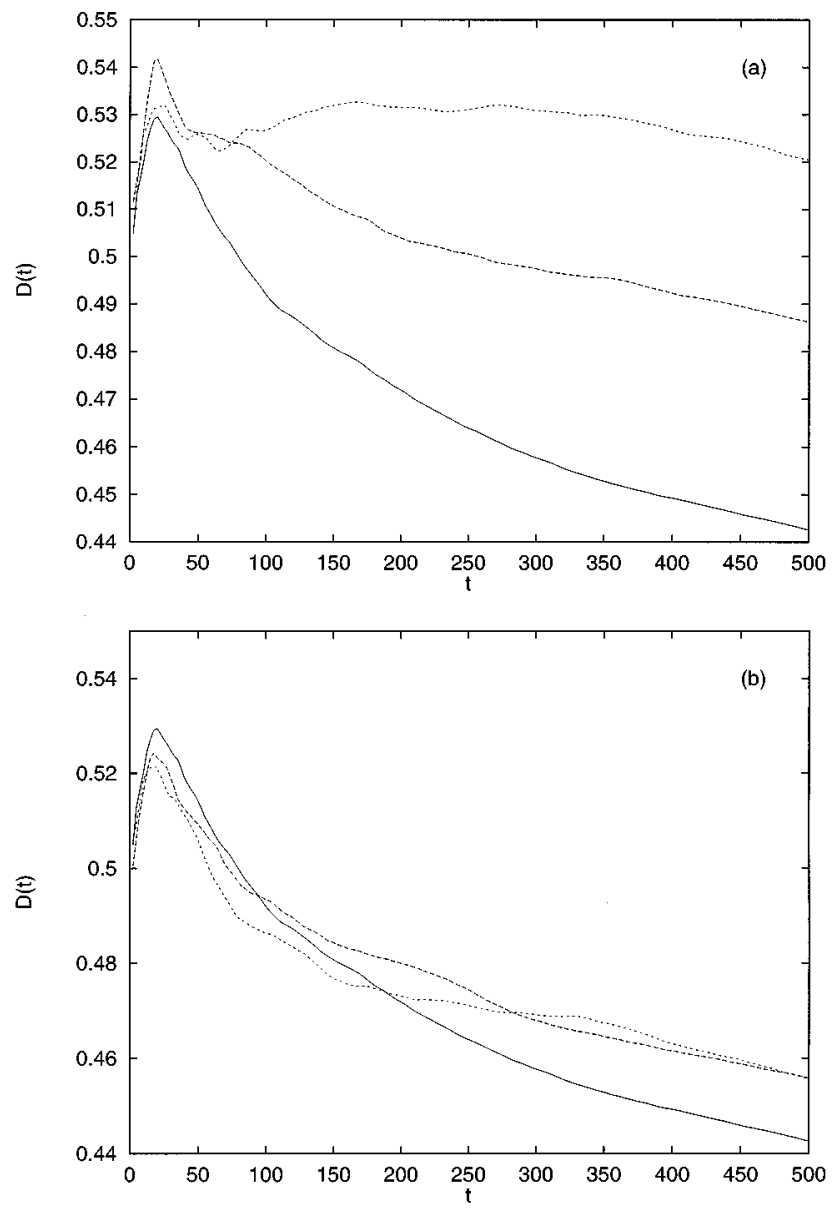

FIG. 6. $D(t)$ for constant $u_{0}^{2}=0.0249$, varying both $\epsilon_{0}$ and $\lambda$ (solid lines: $\epsilon_{0}=10, \lambda=2$, long dashes: $\epsilon_{0}=24.4141, \lambda=2.5$, short dashes: $\epsilon_{0}=50.6250, \lambda=3$ ). (a) For constant viscosity $\nu=1$. One observes a transition of diffusional to anomalous behavior with growing $\lambda$. (b) Varying also $\nu$, keeping thus constant both $u_{0}^{2}$ and $t_{0}=4$. Here the above transition is not observed; on the opposite, results are very similar (taking statistical error into account). In (b), solid line: $\nu=1$, long dashes: $\nu=1.5625$, and short dashes: $\nu=2.25$. The other parameters mentioned are as in Fig. 4 .

Fig. 6(b) we do not only not observe such a transition, but find also that all the results are similar. This means that for the temporal development of $D(t)$ the correlation time plays a much more important role than the correlation length of the flow. This was not so in the case of $L(t)$, which depended very little on $t_{0}$ for constant $u_{0}^{2}$. Clearly, intensity and correlation time are here of similar importance.

\section{SUMMARY AND DISCUSSION}

In this paper we discussed diffusion in a dynamically evolving, phase-separating system with convection. Based on the results of I, we were mainly interested in the question of whether the earlier found prolonged anomalous diffusional process was able to stand up against turbulent mixing. Realizing that in our model the scalar variable $\psi$, or the density of the diffusing substance $C$, couples both directly and indirectly (via the binary medium $\chi$ ) to the flow, we first discussed possible effects of the mixing on $\chi$.

In I we gave evidence that the "mechanism," responsible for the ("infinitely") long anomalous diffusion in such a 
system, was what we named the "reservoir effect" with its appropriate measure being the total interface area. Quite naturally then, upon including the turbulent flow we concentrated on its effect on the latter quantity. In [7] it had been shown that there exists a threshold or critical $\lambda_{c}$, such that for $\lambda>\lambda_{c}$ the structures cease to grow. In this paper we gave further evidence of this fact, showing that this is a continuous process depending on the mixing efficiency. From physical reasoning we know that the latter must be antiproportional to the eddy size (or correlation length of the flow) and proportional to the correlation time. Calculating $L(t)$ for different values of $\lambda$ and $\nu$ this was confirmed. Varying $\epsilon_{0}$ and $\lambda$ for constants $u_{0}^{2}$ and $\nu$ resulted in only small differences in the results. This indicates that the most important parameter here is the intensity of the flow, though a certain dependency on the correlation time exists.

Turning now to the evaluation of $D(t)$, we found that turbulent mixing very effectively facilitates matter interchange, such that even for smaller intensities of the flow, a purely diffusional regime is reached in medium time. In fact, the mixing is so effective (depending naturally on the relative weight, i.e., on $D^{*}$ and $u_{0}^{2}$ ) that by far the dominant coupling is the direct one. The changes in the temporal behavior of the total interface area are by far not sufficient to explain the distinct behavior with and without flow. On the other hand, neglecting the latter, i.e., dropping the coupling of $\chi$ to the flow, resulted in very similar results compared with those where all couplings were included.

Apart from the competition between the two distinct mechanisms of matter transport, the concept of mixing efficiency is central to explaining both the dependencies of $L(t)$ and $D(t)$. So, it was found that $D(t)$ varies in the same manner as $L(t)$ with $\nu$. Up to the time calculated $(t=500)$ we found transitions from anomalous to diffusional behavior upon diminishing $\nu$ (or $\lambda$ ). On the other hand, we found that in the case of $D(t)$ both fundamental parameters $u_{0}^{2}$ and $t_{0}$ are of similar importance. Keeping constant both intensity and correlation time of the flow, very similar results were found, indicating that there is little or no dependence on the correlation length (within the range of correlation lengths used). We suspect that the asymptotic value of $D(t)$ depends on $u_{0}^{2}$ and $t_{0}$.

This result is interesting if looked at from the viewpoint of the distribution of energy. The intensity of the flow appears herein as the integral over the wavelength of said spectrum, while the correlation time does not appear at all in the equilibrium distribution. Reformulating the results in this context seems to indicate that the distribution of the energy on the different wavelengths involved plays a minor role compared to its intensity and dissipation. It would be interesting to investigate whether similar results as the ones shown here could be obtained by a single mode flow.

Finally let us remark on the generality of our model chosen here. From our viewpoint, this model has two major drawbacks. The medium used (i.e., the mixture of the substances $A$ and $B$ ) is characterized only by different bulk diffusion coefficients. Up to now, we assumed (as in the majority of articles published in this area) equal viscosities for both phases. Naturally, this is a rather harsh restriction. In the future we would like to include a $\chi$-dependent (and therefore space-dependent and time-dependent) viscosity. First attempts have revealed the complexity of this problem. Secondly, we have been dealing with binary alloys, thus completely neglecting the hydrodynamical modes of a more complete ansatz that would be applicable to fluids also. Farrell and Valls have stressed in a series of papers (see $[17,18]$ and references therein) the importance of currents in the growth dynamics. As the currents affect the growth rate in a relevant manner (they find-depending on the modelexponents $\left[R(t) \propto t^{n}\right] n$ of up to 0.69 as opposed to $1 / 3$ of the Lifshitz-Slyozov time law) it is most interesting to investigate the influence of said current(s) in diffusion in inhomogeneous media. Both subjects are now under investigation.

\section{ACKNOWLEDGMENTS}

We gratefully acknowledge financial support by the Deutsche Forschungsgemeinschaft and by the Generalitat de Catalunya under the CIRIT-PIEC program. Computer time provided by the Center de Supercomputació de Catalunya is also acknowledged.
[1] H. Lehr, F. Sagués, and J. Sancho, Phys. Rev. E 54, 5028 (1996).

[2] A. Careta, F. Sagués, and J. Sancho, Phys. Fluids 6, 349 (1994).

[3] A. Careta, F. Sagués, L. Ramirez-Piscina, and J. Sancho, J. Stat. Phys. 71, 235 (1993).

[4] A. Careta, F. Sagués, and J. Sancho, Phys. Rev. E 48, 2279 (1993).

[5] A. Martí, A. Careta, F. Sagués, and J. Sancho, Phys. Fluids 9, 1078 (1997).

[6] J. Aronowitz and D. Nelson, Phys. Rev. A 29, 2012 (1984).

[7] A.M. Lacasta, J.M. Sancho, and F. Sagués, Phys. Rev. Lett. 75, 1791 (1995).

[8] C. Taylor and J. Cahn, J. Stat. Phys. 77, 183 (1994).

[9] J. Cahn and A. Novickcohen, J. Stat. Phys. 76, 877 (1994).
[10] S.C. Glotzer, D. Stauffer, and N. Jahn, Phys. Rev. Lett. 72, 4109 (1994).

[11] C.C. Ko, T. Kyu, and S. Smith, J. Polym. Sci. B 33, 517 (1995).

[12] A. Vasishta, Chem. Eng. Commun. 129, 29 (1994).

[13] J.M. Liu, Z.G. Liu, and Z. Wu, Chin. Phys. Lett. 11, 634 (1994).

[14] R. Kraichnan, Phys. Fluids 13, 22 (1970).

[15] A. Kolmogorov, C. R. Acad. Sci. 30, 301 (1941).

[16] L.O. Landau and E.M. Lifshitz, VIII: Electrodynamics, A Course in Theoretical Physics (Pergamon Press, Oxford, 1980).

[17] J. Farrell and O. Valls, Phys. Rev. B 40, 7027 (1989).

[18] O. Valls and J. Farrell, Phys. Rev. E 47, R36 (1993). 